\title{
An Information-Theoretic Framework for Joint CS-ICA Recovery of Sparse Biosignals
}

\author{
Yahia Alghorani, Member, IEEE, and Salama S. Ikki, Senior Member, IEEE
}

\begin{abstract}
The aim of this study is to propose an information-theoretic framework for compressed sensing (CS)-independent component analysis (ICA) algorithms that can be used for the joint recovery of sparse biosignals. The proposed framework supports real-time patient monitoring systems that enhance the detection, tracking, and monitoring of vital signs remotely via wearable biosensors. Specifically, we address the problem of sparse signal recovery and acquisition in wearable biosensor networks, where we present a new analysis of CS-ICA algorithms from an information theory perspective to compute the sampling rate required to recover sparse biosignals corrupted by motion artifacts and interference, which to the best of our knowledge, has not been studied before. Our analysis and examples indicate that the proposed approach helps to develop low-cost, low-power edge computing devices while improving data quality and accuracy for a given measurement. We also show that, under noisy measurement conditions, the CS-ICA algorithm can outperform the standard CS method, where a biosignal can be retrieved in only a few measurements. By implementing the sensing framework, the error in reconstructing biosignals is reduced, and a digital-to-analog converter operates at low-speed and low-resolution.
\end{abstract}

Index Terms—Remote patient monitoring systems, telemedicine, joint sparse recovery for biosignals, wearable biosensor networks.

\section{INTRODUCTION}

$\mathrm{R}$ ECENT developments in patient monitoring systems have led to important applications in the field of biomedical engineering. Wearable devices integrated with cyber-physical systems (CPS) make the medical monitoring of patients with chronic conditions easier and more consistent, allowing clinicians and caregivers to supervise their patients remotely and providing feedback to help maintain an optimal health status, regardless of patient location. These systems are being increasingly used in hospitals and clinics to provide continuous high-quality care to patients in complex clinical scenarios [1].

Recent advances in wearable sensors make CPS a powerful candidate for real-time e-health monitoring, extending to different monitoring areas, including homes, buildings, means of transport, etc. Unlike traditional embedded systems, CPS is typically designed as a network of real-time embedded computing interaction with physical elements that can ensure the adaptability, autonomy, reliability and functionality of wearable biosensor networks. An essential part of CPS is the internet of things (IoT) edge computing platforms, which can enable smart mobile healthcare services, through which patient data is collected by wearable biosensors and edge computing devices (e.g., smart watches, mobile devices) to be transferred to ambient sensors and then stored in medical servers for monitoring health status. The IoT edge computing platform consists of a large number of real-time edge devices (data aggregators) that collect large amounts of data from patients in different locations and make them accessible to clinicians at any time for analysis (see, e.g., Fig.1).

- Y. Alghorani and S.ikki are with the Department of Electrical Engineering, Lakehead University, Thunder Bay, On, Canada (e-mail: yahia.alghorani@ieee.org; sikki@lakeheadu.ca).
Big data analytics for CPS help clinicians predict illness, prepare diagnosis, and plan treatment, resulting in overall improved quality of care and reduced cost. By exchanging medical records for patients between public and private hospitals, doctors and specialists would be able to predict where the patient is located on the spectrum of disease progression more accurately and efficiently. Managing and monitoring vital signs [2], such as heart and respiratory rates, blood pressure, blood flow, blood glucose, body temperature, oxygen saturation, electroencephalogram (EEG), electrocardiogram (ECG), requires wearable sensing platforms that can capture vital signs and biometrics, and deliver data from the patient to the edge device, as well as to the CPS cloud, for medical analysis.

The main challenge is to implement remote monitoring and tracking of patients in sensors and data acquisition/detection, i.e., when vital signs (biosignals) contain noise and artifacts. For example, ECG signals are affected by patient motion and they often suffer from low signal-tonoise ratio (SNR) due to motion artifacts and interference effects. Artifacts can be defined as distorted signals caused by internal or external sources (e.g., muscle movement [3], overlapping of data transmission where inter-biosensor interference occurs within the same wearable biosensor network [4-6]) or inter-network interference where the wearable biosensor network interferes with other nearby wireless sensor networks operating in the industrial, scientific, and medical (ISM) radio bands [7]. Wearable biosensors generate large amounts of patient data that contain motion artifacts and interference that can distort vital signs and reduce detection accuracy, resulting in inadequate diagnosis and treatment. Furthermore, due to continuous patient monitoring, the high-power consumption of edge computing devices poses another major challenge for researchers to adopt such systems in daily use. 


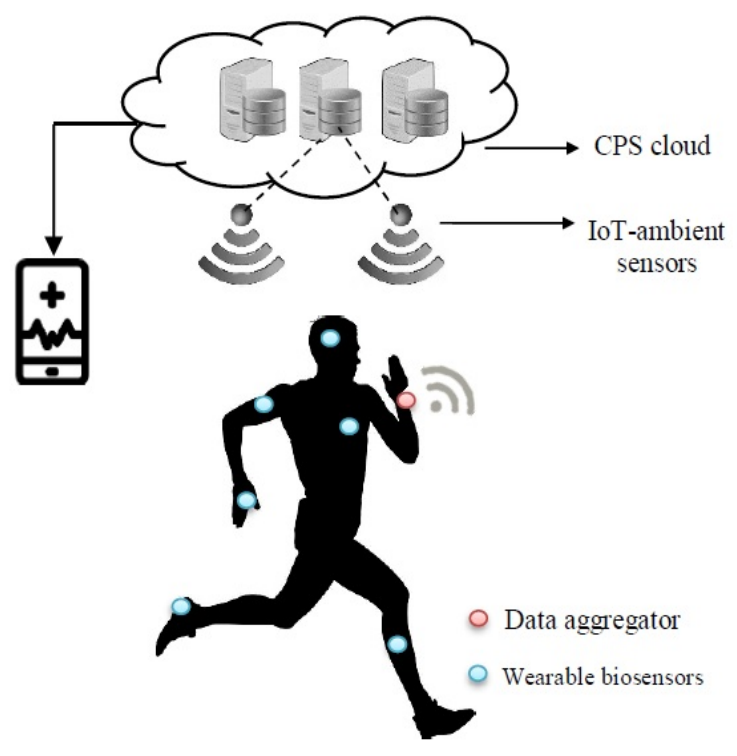

Fig. 1: IoT edge computing platform for continuous patient monitoring, where vital signs are collected through wearable biosensors and then send to the edge device/data aggregator (e.g., smart watch, mobile device) which in turn transmits data flows to ambient sensors and medical servers for analysis

From a physical layer perspective, the presence of motion artifacts and interference in biosignals requires an increase the number of measurements/samples needed by conventional compressed sensing (CS) methods [8] to improve the quality of the reconstructed biosignals, making the speed and resolution of sampling devices, such as digital-toanalog converters (DACs) and analog-to-digital converters (ADCs), high, i.e., high-cost hardware implementation and power consumption of patient monitoring systems. With a complete set of $n$ discrete-time samples of a sparse biosignal, the design of high-speed sampling devices becomes more complicated for edge computing devices, leading to large energy consumption due to continuous monitoring. In other words, the presence of motion artifacts and interference in the sparse biosignals requires an increase in the number of measurements needed by CS to improve the quality of the reconstructed image/signal, making the computational requirements (i.e., sampling rate, resolution) of sampling devices increase to improve the detection accuracy of sparse biosignals [8], [9]. Therefore, the goal of this work is to design and implement a low-complexity computation algorithm that can eliminate noise and artifacts in wearable biosensor networks at a low-cost hardware implementation and power consumption. In order to reduce the number of noisy biosignal measurements and obtain a high-resolution biosignal, we perform independent component analysis (ICA) [10]. ICA is a computational method that separates a multivariate signal into independent non-Gaussian signals, and is mainly used to remove artifacts from EEG recordings. The primary driving force behind the use of ICA in patient monitoring is that solutions are required to address the data sparsity ${ }^{1}$ problem in the presence of motion artifacts and interference. Due to biosignal artifacts, high-resolution and high bit-depth DACs/ADCs are needed to restore biosignals. Since ICA recovery algorithms are powerful tools even

1. Sparsity is defined as having a small number of coefficients that capture most of the information contained in a biosignal. in noisy environments, we aim to use these algorithms to remove noise and artifacts from sparse biosignals, so that the CS can reduce the number of measurements (i.e., sampling rate) needed to retrieve viable biosignals and use low-speed and low-resolution sampling devices, i.e., lowcost implementation and power consumption of patient monitoring systems.

\section{Related Work}

\section{$2.1 \mathrm{CS}$}

In recent years, there has been a research focus on CS applications in wireless body sensor networks and telemonitoring purposes (see, for example [11-14]), where the CS hardware architecture has been divided into two main methods: analog and digital CS. For instance, in [11], the digital CS method (where the linear CS compression is applied after the ADC) was used to recover fetal ECG signals. In [15] and [16], the analog CS has been applied to ECG, where the compression occurs in the analog sensor readout electronics prior to the ADC. Despite the fact that the analog CS method reduces the cost and power consumption of sampling devices compared with the digital CS method, its demonstration still requires extensive work on the analog sensor read-out electronics. Hence, in the current work, we propose to use the digital CS (as described in Fig.2).

\subsection{ICA}

In medical applications, it is common practice to use the digital ICA algorithm to restore EEG signals (where the ADCs are located at the receiver and data is processed in the digital domain [17], [18]). This type of algorithm requires highspeed sampling rate (due to the use of full measurements: $m \geq n)$ to find the independent components/EEG signals, resulting in high-cost and high-power devices. On the other hand, few studies have discussed the analog VLSI implementation of ICA algorithms [see [19] for intelligent hearing aid applications] where the low-energy ICA architecture has been proposed in a noisy and reverberant environment, and experiments have shown a clear separation and precise localization of two speech sources.

However, both CS and ICA methods have some limitations when used alone, and pre/post-processing techniques must be adopted. Some studies have recently proposed new frameworks for the joint CS-ICA recovery to remove artifacts from EEG signals; see, e.g., [20-22], where the CS method is preceded by the ICA data processing method to reduce power consumption of the sensing unit (wearable biosensor network). It is noteworthy that the implementation of ICA prior to CS can lead to large power consumption at the sensing unit and would not be suitable for telemedicine applications. This is because the ICA algorithm is computationally intensive, and such an operation must be performed on the edge device. [23], [24].

There are other recent studies that have discussed this issue (see, e.g., [25-27]), where the ICA is performed after the CS. In fact, there are major drawbacks of this method due to the neglect of two important factors: 1) The use of a Gaussian random measurement matrix $\Phi \in R^{m \times n}$ in the sensing unit to measure sparse biosignals usually 


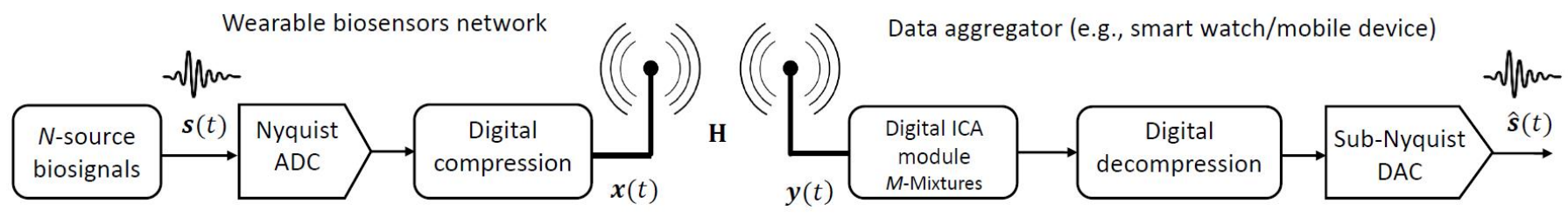

Fig. 2: Proposed Framework

following non-Gaussian distributions (e.g., sub-Gaussian, super-Gaussian, or mixed super-, sub-, and Gaussian distributions) [28]. Most of the measurement matrices discussed in the CS theory are based on impractical assumptions due to the Gaussianity of the measurement matrix and the strict sparsity of the input vector $x \in R^{n}$ [29]. However, in wireless communications (where wearable biosensors suffer from motion artifacts and interference, and operate under non-Gaussian input conditions), we cannot assume that the measurement matrices are Gaussian and random; therefore, the measurement matrices of CS must be performed on the edge device after the ICA algorithm. 2) Increased power consumption of the sensing unit as the number of noisy measurements $(m)$ increases in the measurement matrix $\Phi$ due to motion artifacts and noise interference that exist in sparse biosignals. The presence of noise and interference in wearable biosensor networks requires increasing the number of measurements by CS to improve the signal/image quality [9], which means increased computational complexity and power consumption of the sensing unit.

In order to bridge the gap between the CS and ICA methods in motion artifacts and interference scenarios, we provide an efficient co-design platform for CS-ICA algorithms that offers significant energy savings compared to other state-of-the-art methods, where we introduce a new analytical framework for CS-ICA algorithms from an information theory perspective, which helps obtain the sampling rate $\left(f_{s}\right)$ required to recover the sparse biosignals from noisy measurements, which to the best of the authors' knowledge, has not yet been explicitly identified in the literature. The proposed sensing framework (described in Fig.2) enables efficient implementation in CS hardware and provides valuable guidelines for communications engineers/researchers working on the design of sparse recovery algorithms for remote patient monitoring systems, by choosing the appropriate sampling device requirements (e.g., sampling rates and resolutions for DACs). Specifically, the paper's contributions are summarized as follows:

- Developing low-cost, low-power edge computing devices through digital CS-ICA algorithms, where we address the sparse signal recovery problem in wearable biosensor networks, and reconstruct the corrupted sparse biosignal due to motion artifacts and interference at the sampling rate $f_{s} \geq 2 \frac{\mathrm{m}}{T}$, where $m \geq 2 \log _{2}\left(\frac{n}{K}\right) / \log _{2}(1+M . S N R)$ samples.

- Unlike conventional CS recovery methods, the CSICA algorithms reduce biosignal reconstruction errors and provide a low mean squared error compared to standard CS methods (where the noise power in the collected data is reduced to up $\frac{\epsilon}{M}$ when reconstructing a sparse biosignal).
TABLE I

NOTATION

\begin{tabular}{|c|c|}
\hline$K$ & $\begin{array}{l}\text { Small number of non-zero elements (coefficients) that capture } \\
\text { most of the information contained in a biosignal. }\end{array}$ \\
\hline$n$ & Number of coefficients/samples in a biosignal \\
\hline$m$ & Number of measurements/samples for a biosignal \\
\hline$N$ & Number of wearable biosensors \\
\hline$M$ & Number of sensors for the data aggregator, $M \geq N$. \\
\hline$s_{r}$ & $s_{r}=\left[s_{1}, \ldots, s_{n}\right]$ denotes the $r$-th source biosignal of size $n$ \\
\hline$\Psi_{n \times n}$ & $\begin{array}{l}\Psi_{n \times n} \text { is a unitary matrix that can discard the small coefficients } \\
\text { of } s_{r}\end{array}$ \\
\hline$x_{r}$ & $\begin{array}{l}x_{r} \text { denotes the } r \text {-th sparse biosignal of size } n \text {, defined as } x_{r}= \\
\Psi_{r} S_{r}\end{array}$ \\
\hline$\Phi_{r}$ & The measurement matrix, $\Phi_{r} \in R^{m \times n}, n \gg m \gg K$ \\
\hline$A_{r}$ & The sensing matrix, defined as $A_{r}=\Phi_{r} \Psi_{r}, A_{r} \in R^{m \times n}$ \\
\hline$h_{r}$ & $\begin{array}{l}\text { Constant channel vector for the } r \text {-th source biosignal, } h_{r} \in \\
R^{M \times 1}\end{array}$ \\
\hline$w_{r}$ & The digital combiner, $w_{r} \in R^{1 \times M}$ \\
\hline$n_{r}$ & Additive white Gaussian noise for the $r$-th sparse biosignal \\
\hline$y_{r}$ & Receiving signal for the $r$-th biosensor \\
\hline$\hat{x}_{r}$ & The estimated sparse biosignal, defined as $\hat{x}_{r}=w_{r} y_{r}$ \\
\hline $\mathrm{H}$ & The unknown mixing matrix of source biosignals, $\mathrm{H} \in R^{M \times N}$ \\
\hline $\mathrm{W}$ & The unmixing matrix, defined as $\mathrm{W}=\mathrm{H}^{T}, \mathrm{~W} \in R^{N \times M}$ \\
\hline$\left\|s_{r}\right\|_{l_{1}}$ & Standard $l_{1}$ norm of $s_{r},\left\|s_{r}\right\|=\sum_{k=1}^{n}\left|s_{r, k}\right|$ \\
\hline$\left\|s_{r}\right\|_{l_{2}}$ & Standard $l_{2}$ norm of $s_{r},\left\|s_{r}\right\|^{2}=\sum_{k=1}^{n}\left|s_{r, k}\right|^{2}$ \\
\hline$H\left(\hat{X}_{i}\right)$ & $\begin{array}{l}\text { Differential entropy of the } i \text {-th sparse biosignal } \hat{X}_{i} \text {, defined in } \\
\text { (8). }\end{array}$ \\
\hline
\end{tabular}

\section{Problem Formulation}

In order to develop an energy-efficient sensing framework for patient monitoring systems, we outline the steps of CS used in noisy measurements, where source biosignals are sparse in the time domain (i.e., the $K$-source biosignal vector $s_{K}$ contains $K$ non-zero elements and satisfies $\|s\|_{l_{0}} \leq K \ll n$ ). If the source biosignal $s \in R^{n}$ is not sparse, we can make it so through CS, using an inverse discrete cosine transform (IDCT) matrix to produce a sparse vector; that is, $x=\Psi s$, where $\Psi_{n \times n}$ is a unitary matrix that can discard the small frequency coefficients of $s$ due to motion artifacts being measured by a motion sensor (accelerometer), i.e., many frequency coefficients are set to zero via $\Psi$ after adding a quantization step to the IDCT. With CS, we can employ low-speed DACs (i.e., sub-Nyquist sampling rates) to recover the sparse biosignals and reduce the power consumption and cost of edge devices/data aggregators. 


\subsection{CS Acquisition}

In telemedicine applications and e-health systems, where motion artifacts and inter-biosensor interference can occur in the $N$-wearable biosensor network (see, e.g., Fig.3), the receiving sparse signal for each biosensor, $y_{r} \in R^{M \times n}$, $r \in\{1,2, \ldots, N\}$, at the $M$-sensor array (mixtures) of data aggregator (where $M \geq N$ ), is expressed as

$$
y_{r}=\sum_{i=1}^{N} h_{r} x_{i}^{T}+n_{r}
$$

where $x_{i}=\Psi_{i} s_{i}$ represents $n$-pixel image/video/radio pulses, which are corrupted by additive white Gaussian noise (AWGN) $n_{r} \in R^{M \times n}, h_{r} \in R^{M \times 1}$ is a constant channel vector with array elements $h_{r j}$, which depends on the distance between the $r$-th biosensor and the $j$-th sensor node of the data aggregator. The received signal is then processed by a digital combiner $w_{r} \in R^{1 \times M}$ to obtain $\hat{x}_{r}=w_{r} y_{r}$, which can be rewritten as

$$
\hat{x}_{r}=\underbrace{w_{r} h_{r} x_{r}^{T}}_{\text {desired signal }}+\underbrace{\sum_{i \neq r}^{N} w_{r} h_{r} x_{i}^{T}}_{\text {artifacts and interference }}+\underbrace{w_{r} n_{r}}_{\text {noise }},
$$

where $w_{r}=h_{r}^{T}$. After extracting the desired sparse biosignal, $\hat{x}_{r}$, and using the CS, the received signal is expressed as $z_{r}=\Phi_{r} \hat{x}_{r}$, namely

$$
z_{r}=A_{r} s_{r}+v_{r}
$$

where $z_{r} \in R^{m}$ is the measurements vector for the biosensor $r, A_{r}=\Phi_{r} \Psi_{r}$ is the sensing matrix, $\Phi_{r} \in R^{m \times n}$ is the measurement matrix (where $n \gg m \gg K$ ), and $v_{r} \in R^{m}$ is the effective noise vector that contains the motion artifact, interference, and noise component. Here, the sensing matrix $A_{r} \in R^{m \times n}$ obeys the the restricted isometry property (RIP), i.e.,

$$
\left(1-\delta_{K}\right)\left\|s_{r}\right\|_{l_{2}}^{2} \leq\left\|A_{r} s_{r}\right\|_{l_{2}}^{2} \leq\left(1+\delta_{K}\right)\left\|s_{r}\right\|_{l_{2}}^{2}, \delta_{K} \in(0,1),
$$

when $\delta_{K}$ is not too close to one and its entries are drawn from a suitable distribution, e.g. a Gaussian distribution [9]. So, if the RIP of order $K$ is established, then it is sufficient to have $m=O\left(K \cdot \log _{2} n / K\right)$ samples.

\subsection{CS Recovery}

In order to recover $s_{r}$ from $z_{r}$, we consider the following optimization problem

$$
\min _{s_{r}}\left\|s_{r}\right\|_{l_{1}} \text { s.t }\left\|A_{r} s_{r}-z_{r}\right\|_{l_{2}} \leq \epsilon,
$$

where $\left\|v_{r}\right\|_{l_{2}} \leq \epsilon$ is the maximum noise power, $\left\|s_{r}\right\|_{p}$ stands for the standard $l_{p}$-norm and $\left\|s_{r}\right\|_{l_{0}}$ counts the number of nonzero elements in $s_{r}$. If we assume that $\delta_{2 K}<\sqrt{2}-1$, the solution to the convex problem above is satisfied by

$$
\left\|\hat{s}_{r}-s_{r}\right\|_{l_{2}} \leq C_{0} \cdot\left\|s_{r}-s_{r, K}\right\|_{l_{1}} / \sqrt{K}+C_{1} . \epsilon,
$$

where the constants $C_{0}$ and $C_{1}$ are typically small [30, eq. (14)]. The result in (5) indicates that the noise power in the data can significantly increase the reconstruction errors, which means that we need more measurements ( $m$-samples) to recover the source biosignal vector $s_{r}$. An equivalent expression has been determined by [31], $\left\|\hat{s}_{r}-s_{r}\right\|_{l_{2}}<$ $\left\|v_{r}\right\|_{l_{2}}+\epsilon / 1-\delta_{2 K}$.

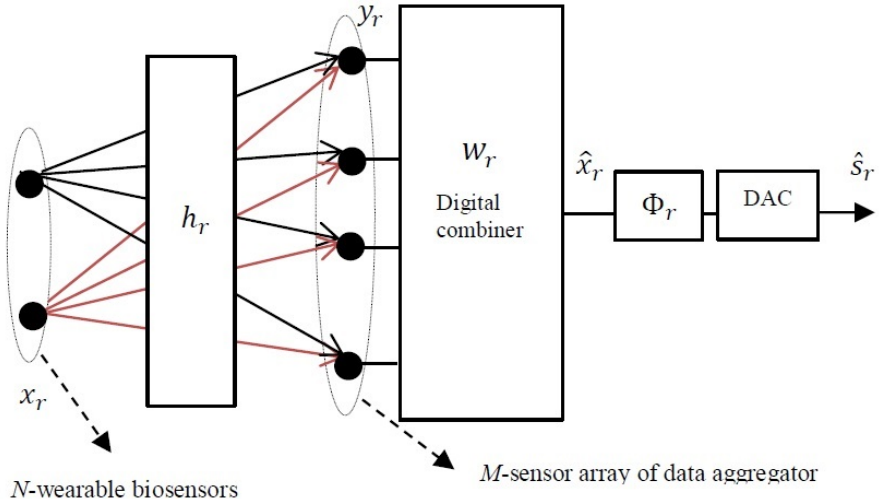

Fig. 3: Artifacts and inter-biosensor interference scenarios where motion artifacts are monitored by the motion sensor and $N$ sparse biosignals are retrieved through standard digital CS methods.

Note that when $\delta_{2 K}$ approaches one, the reconstruction process becomes infeasible because of the high error rate. It should be noted that the RIP works well when there is no noise, where the reconstruction of the biosignal is more accurate (i.e., $\left\|\hat{s}_{r}-s_{r}\right\|_{l_{1}} \leq C_{0} .\left\|s_{r}-s_{r, K}\right\|_{l_{1}}$, [9, eq. (10)]). Although the CS technique resolves the sparse data acquisition problem, there are still some conditions and limitations that should be considered when dealing with scenarios of motion artifacts and interference (i.e., it is impossible to construct the sparse vector $s_{r}$ when the effective noise level, $v_{r}$, is high). Therefore, the precise calculation of the digital combiner elements $w_{r}$ is important to reduce the high noise level of $v_{r}$ so that the biosignal is retrieved in relatively few measurements.

\section{Proposed Framework}

ICA was originally developed as a computational method that can be used in various applications including medical signal and image processing (with a non-Gaussian input) [10], where a multivariate biosignal decomposes into independent additive subcomponents without prior knowledge of source biosignals or mixing coefficients. In other words, if we have a mixture of $N$ independent source biosignals, the ICA algorithm works to find the unmixing matrix $\mathrm{W} \in R^{N \times M}$, to extract the source biosignal and remove artifacts from EEG recordings, see Fig.2, where the source biosignals are collected by wearable biosensors and compressed by the digital CS model to be transferred to the data aggregator (e.g., smart watch, mobile device). Here, the ICA scheme is performed to extract the sparse biosignals which are then decompressed by the digital CS model.

Using the joint CS-ICA recovery, we can employ lowspeed and low-resolution DACs (i.e., sub-Nyquist sampling rates and low bit-depths) to restore biosignals and reduce the power consumption and cost of data aggregators. Linear receivers such as zero-forcing and minimum mean squared error equalizers can offer a fair trade-off between performance and complexity. The main drawbacks of these receivers are twofold: 1) Any defects in the estimation of channel and covariance matrices in a large network of biosensors result in residual interference in the data aggregator output, which in turn can lead to performance degradation. 2) There is an increase in the number of iterations needed for 


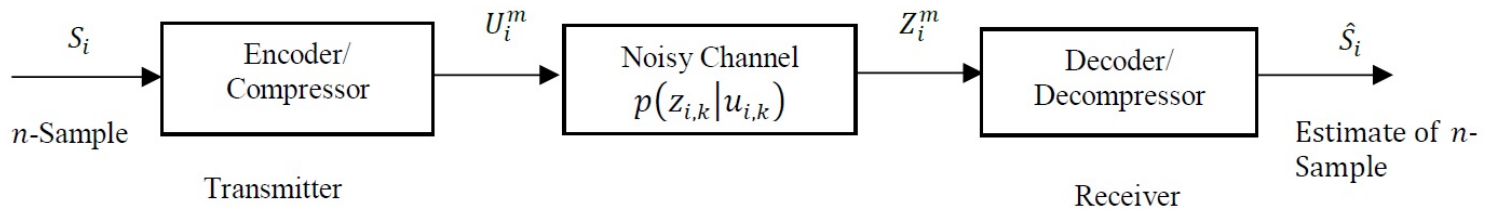

Fig. 4: Block diagram for an $n$-sample compression

convergence. Therefore, the ICA algorithm is suggested as an alternative if the channel state information is unknown, and a fast convergence multi-biosensor detection scheme can be achieved by extracting all independent components directly. Suppose now that the data aggregator observes the mixture signal $y \in R^{M \times 1}$ in the time domain,

$$
y(t)=\mathrm{H} x(t)+n(t),
$$

where $n \in R^{M \times 1}$ is the Gaussian noise vector, $\mathrm{H} \in R^{M \times N}$ is the unknown mixing matrix of source biosignals $s \in$ $R^{N \times 1}$, and $x(t)=\Psi s(t)$ is the sparse vector,

$$
\begin{aligned}
& x(t)=\left[x_{1}(t), \ldots, x_{N}(t)\right]^{T} \\
& s(t)=\left[s_{1}(t), \ldots, s_{N}(t)\right]^{T},
\end{aligned}
$$

where the discrete-time representation of a continuous-time signal, which lies at the heart of analogue to digital conversion [32], defined as $x_{i}(t)=\Psi_{i} s_{i}(t), s_{i} \in R^{n}$ is a source biosignal, and $\Psi_{i} \in R^{n \times n}$ is the unitary matrix generated by the IDCT. We define $t$ as a time index, where $1 \leq t \leq T$.

The digital ICA algorithm estimates the sparse vector $x \in R^{N \times 1}$ using the unmixing matrix $\mathrm{W}$, that is, $\hat{x}(t)=$ $\mathrm{W} y(t)$, where $\mathrm{W}=\mathrm{H}^{T}$ is approached by maximumlikelihood estimation or FastICA algorithms [10]. Furthermore, to eliminate the noise components $\mathrm{Wn} n(t)$, we use an adaptive filter (e.g., Least Mean Square (LMS) methods) to accomplish this task.

Assuming the independence of sparse biosignals $x_{i}$, the joint probability density function (PDF) of continuous random variables $P\left(X_{1}, \ldots, X_{N}\right)$ is factorized as

$$
p_{X_{i}}(x(t))=\prod_{i} p_{X_{i}}\left(x_{i}(t)\right) .
$$

In order to maximize the statistical independence of extracted components (i.e., sparse biosignals), we need to minimize the mutual information of estimated signals $\hat{x}_{i}$ and maximize non-Gaussianity, i.e., $I\left(\hat{X}_{1} ; \ldots ; \hat{X}_{N}\right)=$ $\sum H\left(\hat{X}_{i}\right)-H\left(\hat{X}_{1}, \ldots, \hat{X}_{N}\right)$, where the differential entropy $H\left(\hat{X}_{i}\right)$ of a continuous random variable $\hat{X}_{i}$ with a density function $p\left(\hat{x}_{i}\right)$, is defined as [33, Sec. 8.1]

$$
H\left(\hat{X}_{i}\right)=-\int_{S} p\left(\hat{x}_{i}\right) \log p\left(\hat{x}_{i}\right) d \hat{x}_{i},
$$

where $S$ is the support set of the variable $\hat{X}_{i}$, and the joint differential entropy of multiple continuous random variables $\hat{X}_{1}, \ldots, \hat{X}_{N}$ is defined as [33, Sec. 8.4]

$$
\begin{aligned}
H\left(\hat{X}_{1}, \ldots, \hat{X}_{N}\right)= & -\int p\left(\hat{x}_{1}, \ldots, \hat{x}_{N}\right) \\
& \times \log p\left(\hat{x}_{1}, \ldots, \hat{x}_{N}\right) d \hat{x}_{1} \ldots d \hat{x}_{N} .
\end{aligned}
$$

(9) can also be rewritten as $H\left(\hat{X}_{1} ; \ldots ; \hat{X}_{N}\right) \leq \sum H\left(\hat{X}_{i}\right)$ with equality if $\hat{X}_{1}, \ldots, \hat{X}_{N}$ are independent.

Now let $\hat{X}=\mathrm{W} Y$; using the differential entropy property: $H(\mathrm{~W} Y)=H(Y)+\log |\mathrm{W}|$, we obtain

$$
\begin{aligned}
I\left(\hat{X}_{1} ; \ldots ; \hat{X}_{N}\right) & =\sum H\left(\hat{X}_{i}\right) \\
- & H\left(Y_{1}, \ldots, Y_{M}\right)-\log |\mathrm{W}| .
\end{aligned}
$$

In order to measure the difference in entropy between a given distribution and Gaussian distribution (containing the highest entropy) with the same mean and variance, we use negentropy, defined as $J\left(\hat{X}_{i}\right)=H\left(\hat{X}_{g}\right)-H\left(\hat{X}_{i}\right)$, where $H\left(\hat{X}_{g}\right)$ is the entropy of the Gaussian distribution with unit variance for all estimated sparse biosignals $\hat{x}_{i}$.

In information theory and statistics, minimization of mutual information between multiple random variables $\hat{X}_{i}$ is achieved by minimizing entropy $H\left(\hat{X}_{i}\right)$ or maximizing negentropy $J\left(\hat{X}_{i}\right)$, which is also equivalent to minimizing Gaussianity. Therefore, finding the optimal unmixing matrix $W$ will help to minimize the mutual information between the variables $\hat{X}_{i}$ and make the extracted sparse biosignals uncorrelated (independent) and non-Gaussian. Once the ICA extracts the sparse biosignals with improved SNRs, the CS senses and acquires each sparse biosignal, so the number of measurements $m$ is minimized, and the biosignal is sampled by few measurements at a rate below Nyquist standards.

Suppose the sparse biosignals are independent and identically distributed (i.i.d) random variables (i.e., all sparse biosignals $X_{i}$ have the same probability distribution and are mutually independent), then the sparse biosignal observed by the CS model can be expressed as

$$
z_{i}\left(t_{k}\right)=u_{i}\left(t_{k}\right)+v_{i}\left(t_{k}\right),
$$

where $u_{i}\left(t_{k}\right)=\Phi_{i} \hat{x}_{i}\left(t_{k}\right)$ is the received sparse biosignal vector, $\hat{x}_{i}\left(t_{k}\right)$ is the estimated sparse vector that corresponds to the $i$-th row of $\mathrm{W} y\left(t_{k}\right)$, and $v_{i}\left(t_{k}\right)$ is the $i$ th residual gaussian noise vector after filtering the noise component $\mathrm{Wn} n\left(t_{k}\right)$. Note that $t_{k}, k \in\{1,2, \ldots, m\}$ are the time instants when samples ( $m$-snapshots) are taken.

In order to reconstruct the sparse biosignal $x_{i}$, we fix $\Psi_{i}$ and pick up the input of the $i$-th sampling matrix $\Phi_{i}$ randomly from a non-Gaussian distribution with zero mean and variance $1 / m$ (e.g., sub-Gaussian distribution [34]). Here is typically a flat PDF with a strong tail decay property, so that

$$
E\left[e^{-\lambda u}\right] \leq e^{-\lambda^{2} \rho^{2} / 2}, \text { for all } \lambda \geq 0,
$$

where $E[U]=0, \operatorname{Var}[U] \leq \rho^{2}$, and the sensing matrix $\left[A_{i}=\Phi_{i} \Psi_{i}\right]$ satisfies the RIP condition (i.e., $m \geq$ $c . K \cdot \log _{2} n / K$, where $c$ is a positive constant). Here, the 


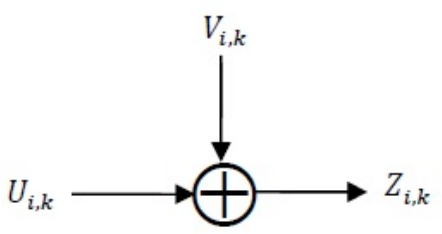

Fig. 5: Gaussian Channel

digital CS-ICA algorithm takes advantage of the RIP property by providing minimum and maximum power for the samples, ensuring that the $K$-sparse vectors do not fall into the null space of the sampling matrix $\Phi_{i}$, which in turn provides a stable recovery for the sparse biosignals.

\section{Generalized Rate of CS-ICA Algorithms}

The sparse recovery algorithm works to reduce motion artifacts and noise interference, where the generalized rate $R_{i}$ of independent sub-Gaussian random variables $U_{i, k} \sim$ $N\left(0, \rho_{i, k}^{2}\right)$, is calculated in the following way:

Proposition. Suppose $U_{i}^{m}=\left(U_{i, 1}, \ldots, U_{i, m}\right) \in U_{i}^{m}$, and $Z_{i}^{m}=\left(Z_{i, 1}, \ldots, Z_{i, m}\right) \in Z_{i}^{m}$ are an $m$-tuple of the random variables $U_{i}$ and $Z_{i}, i \in\{1, \ldots, N\}$, which are generated from the CS and data compression process. Now consider $m$ independent Gaussian channels in parallel, in which we can send the non-Gaussian input $u_{i}^{m}$ through $m$-AWGN channels $v_{i}^{m}$ with power constraint $\frac{1}{m} \sum_{k=1}^{m} P_{i, k} \leq \frac{P}{N}$ and noise variance $\sigma_{i, k}^{2}$. The the digital ICA combiner output is expressed as $z_{i}^{m}=u_{i}^{m}+v_{i}^{m}$ with power gain $\left\|h_{i}\right\|^{2}=$ $\sum_{j=1}^{M}\left|h_{i j, k}\right|^{2}, h_{i}$ is the $i$-th column of the unknown mixing matrix $\mathrm{H}$. Thus, if $U_{i, k}$ and $V_{i, k}$ are independent random variables, then

$$
R_{i}=I\left(Z_{i}^{m} ; U_{i}^{m}\right) \leq \sum_{k} \frac{1}{2} \log _{2}\left(1+\frac{P_{i, k}}{\sigma_{i, k}^{2}} \cdot\left\|h_{i}\right\|^{2}\right),
$$

with equality if $Z_{i, k}$ are independent and sub-Gaussian random variables (i.e., $Z_{i, k} \sim N\left(0, \rho_{i, k}^{2}+\sigma_{i, k}^{2}\right)$ and $\sigma_{i, k}^{2}=$ $\left.E\left[V_{i, k}^{2}\right]\right)$, and the SNR is obtained for the $k$-th sample as $S N R_{i, k}=P_{i, k} / \sigma_{i, k}^{2}$.

Proof. By calling the definition of the information capacity of parallel Gaussian channels [33, Sec. 9.4], we obtain

$$
I\left(Z_{i}^{m} ; U_{i}^{m}\right) \leq \sum_{k} H\left(Z_{i, k}\right)-H\left(V_{i, k}\right) .
$$

Given $H\left(Z_{i, k}\right) \leq \frac{1}{2} \log 2 \pi e\left(\rho_{i, k}^{2}+\sigma_{i, k}^{2}\right), H\left(V_{i, k}\right)=$ $\frac{1}{2} \log 2 \pi e \sigma_{i, k}^{2}$, and $\rho_{i, k}^{2}=P_{i, k} .\left\|h_{i}\right\|^{2}$, (13) can be proven.

\section{Special cases:}

1) Since the ICA algorithm mitigates motion artifacts and interference in wearable biosensor networks, we assume that a) the received SNR is high enough so that we can distribute equal amounts of energy across channels using a water-filling solution (i.e., $P_{i, k} \leq P(N)$, and b) with a coefficient magnitude of $\left|h_{i j, k}\right|^{2}=1$ (i.e, $\left\|h_{i}\right\|^{2}=M$ ). Using Jensen's inequality, (13) can be expressed as

$$
R \leq \frac{1}{2} \log _{2}\left(1+\frac{1}{m} \sum_{k} \frac{P_{i, k}}{\sigma_{i, k}^{2}}\left\|h_{i}\right\|^{2}\right)
$$

$$
\leq \frac{1}{2} \log _{2}(M \cdot S N R),
$$

where $S N R=P / N \sigma^{2}$.

2) On the other hand, assuming that the mixture signal $y$ contains Gaussian and non-Gaussian random variables, we can maximize the entropies $H\left(\hat{X}_{i}\right)$ and minimize the negentropies $J\left(\hat{X}_{i}\right)$, hence finding an ideal unmixing matrix $W$ becomes impossible due to Gaussian increase, leading to a low SNR (e.g., $\log (1+x) \approx x \log _{2} e$ when $x$ is sufficiently small), namely

$$
R \approx \frac{1}{2} M \cdot S N R \cdot \log _{2} e,
$$

where $S N R=P / N \sigma^{2}$.

3) Assuming that the number of measurements is large enough (i.e., $m=n \rightarrow \infty$ ). By the strong law of large numbers, $\operatorname{Pr}\left[\lim _{m \rightarrow \infty} \frac{1}{m} \sum_{k} P_{i, k}=\frac{P}{N}\right]=1$, (13) is calculated as

$$
R \leq \frac{1}{2} \log _{2}(1+M . S N R) .
$$

\subsection{Channel Coding via CS-ICA Algorithms}

To extract the performance gain of digital CS-ICA algorithms, we invoke the channel coding theorem for a discrete memoryless channel [33]. Suppose we connect through a noisy channel $\left(U_{i}^{m}, p\left(z_{i}^{m} \mid u_{i}^{m}\right), Z_{i}^{m}\right)$, where the input samples do not depend on the past output samples, that is to say, $p\left(z_{i, k} \mid u_{i}^{k}, z_{i}^{k-1}\right)=p\left(z_{i, k} \mid u_{i, k}\right) ; p\left(z_{i}^{m} \mid u_{i}^{m}\right)=$ $\prod_{k=1}^{m} p\left(z_{i, k}, u_{i, k}\right)$. The encoder (compressor) $\Psi_{i}$ first takes a block of $n$ samples within $T$ seconds and turns them into a $K$-sparse biosignal, where $\log _{2}\left(\frac{n}{K}\right)$ bits are used to encode/compress the $n$-sample sequence $S_{i}=\left(S_{i, 1}, \ldots, S_{i, n}\right)$, then the decoder (decompressor) does the inverse process. An $\left(2^{m R}, m\right)$ code for the channel $U_{i}^{m}, p\left(z_{i}^{m} \mid u_{i}^{m}\right) Z_{i}^{m}$ consists of a compressing function $U^{m}$ and a decompressing function $Z^{m}$ (see Fig.4). The rate of the code $\left(2^{m R}, m\right.$ ) is defined by $R=\log _{2}\left(\frac{n}{K}\right)$, with a probability of error $P_{e}=\operatorname{Pr}\left(S_{i} \neq \hat{S}_{i}\right)<\epsilon$. So, if we assume that all entries of the source vector $S_{i}$ are i.i.d random variables and the channel coefficients of the samples are defined as $\left|h_{i k}\right|^{2}=1$, then sampling rate for each sparse biosignal is expressed as $f_{s} \geq 2 \frac{m}{T}$, where the number of measurements $m$ required to retrieve the sparse biosignal is calculated as

$$
m \geq \frac{2 \log _{2}\left(\frac{n}{K}\right)}{\log _{2}(1+M \cdot S N R)},
$$

where $\log _{2}\left(\frac{n}{K}\right)=2 H(K / n)=2 K \log _{2}\left(\frac{n}{K}\right)$ bits when $0 \leq$ $K / n \leq 1$. Note that the ICA method brings an additional power gain to the CS method. The higher the SNR, the lower the number of measurements. We can also conclude that

$$
m \geq 2 \log _{2}\left(\frac{n}{K}\right) / \log _{2}(M . S N R),
$$

when the number of ICA mixtures is very large i.e., $M \rightarrow$ $\infty$, where $S N R=P / \sigma^{2}$. By assuming that the sensing matrix $A_{i}$ satisfies the RIP property, $\left(1-\delta_{i, K}\right)\left\|s_{i}\right\|_{l_{2}}^{2} \leq$ $\left\|u_{i}\right\|_{l_{2}}^{2} \leq\left(1+\delta_{i, K}\right)\left\|s_{i}\right\|_{l_{2}}^{2}$, where $u_{i}=\left\|h_{i}\right\|^{2} A_{i} s_{i}$, then 


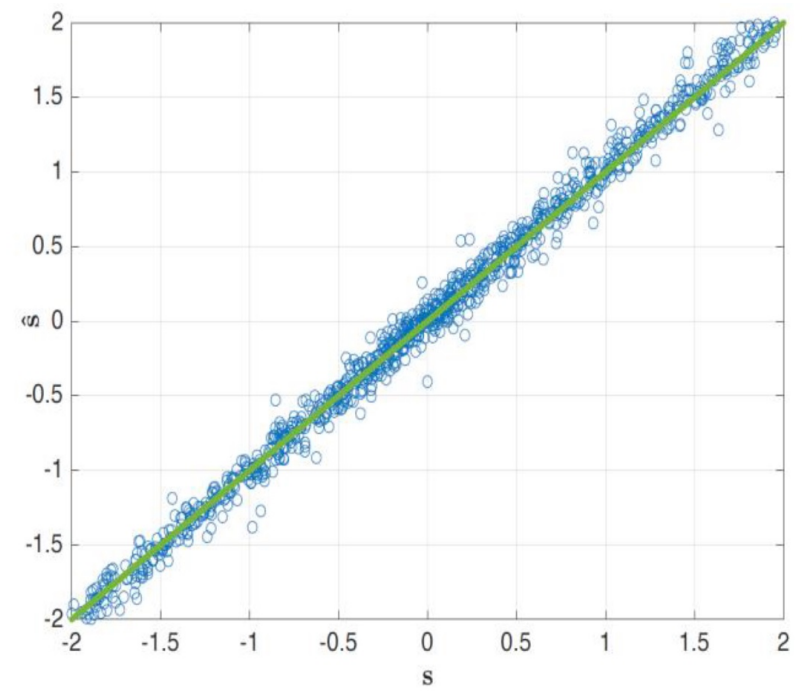

(a)

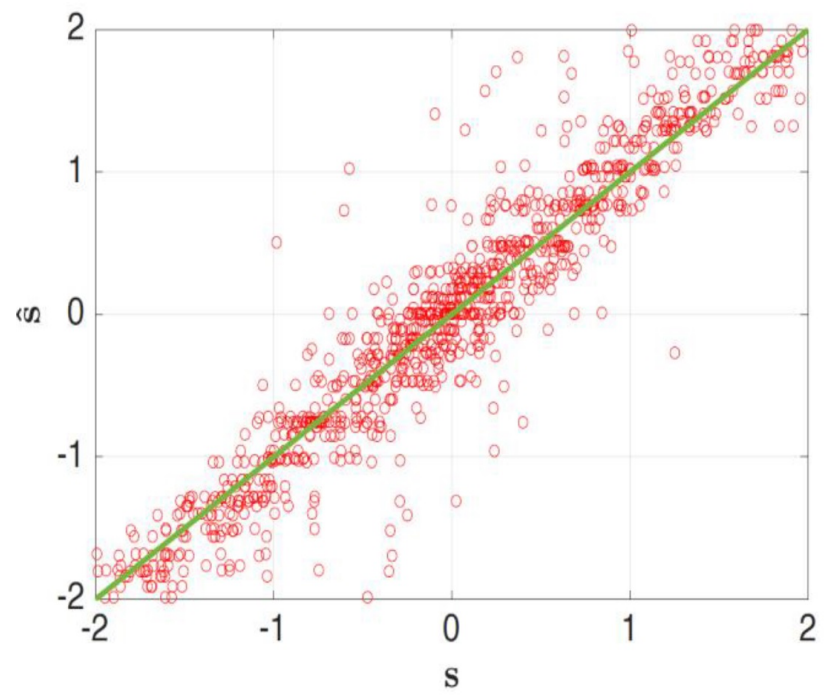

(b)

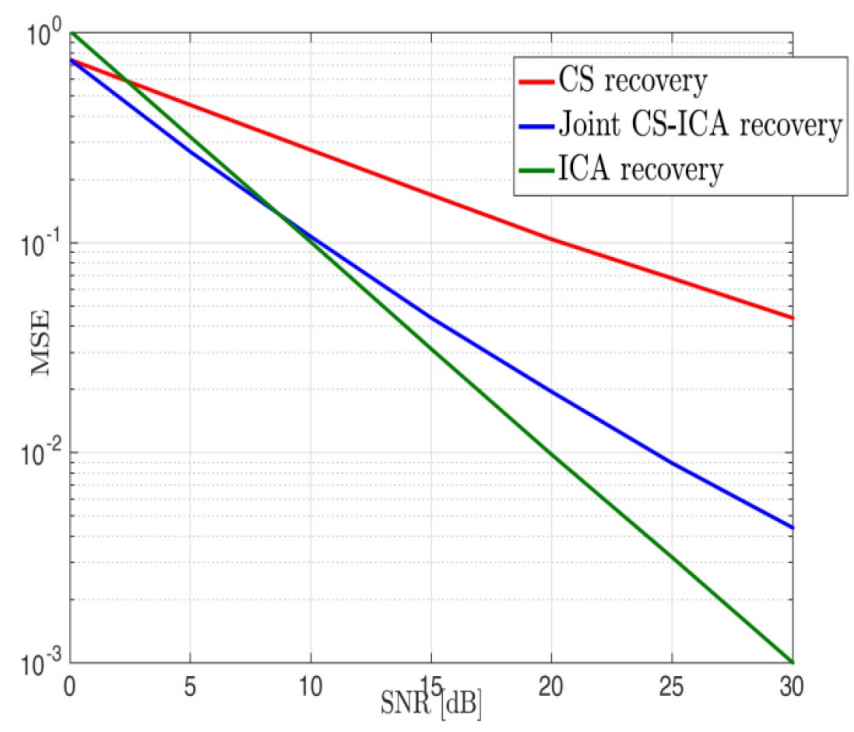

(c)

Fig. 6: The biosignal reconstruction errors for both CS-ICA (a) and CS (b) methods, where $N=2, M=2$. (c) Comparison of the mean squared error for various sparse biosignal recovery methods, where the sparse biosignal is recovered at $n=1000, m=300$ and different SNR values. the receiver can reconstruct the $n$-sample sequence, $S_{i}=$ $S_{i, 1}, \ldots, S_{i, n}$, in the digital domain by solving the convex problem

$$
\hat{s}_{i}=\min _{s_{i} \in R^{n}}\left\|s_{i}\right\|_{l_{1}} \quad \text { s.t. }\|\| h_{i}\left\|^{2} A_{i} s_{i}-z_{i}\right\|_{l_{2}} \leq \epsilon .
$$

By using the triangle inequality [34] and the theorem in (5) for $\left\|v_{i}\right\|_{l_{2}} \leq \epsilon=\sigma_{i}^{2}$, the solution to (16) obeys

$$
\left\|\hat{s}_{i}-s_{i}\right\|_{l_{2}} \leq C_{i, 0} \cdot\left\|s_{i}-s_{i, K}\right\|_{l_{1}} / \sqrt{K}+C_{i, 1} \sigma_{i}^{2} /\left\|h_{i}\right\|^{2}
$$

From (17), we conclude that the digital CS-ICA algorithm can reduce the effects of motion artifacts and interference on wearable biosensor networks, recover sparse biosignals with high quality, allowing the edge device/data aggregator to use low-speed/low-resolution DACs to restore these sparse biosignals.

\section{Performance Analysis}

In order to gain further insight into the performance of digital CS-ICA algorithms, we display the error in retrieving sparse biosignals from noisy data as shown in Fig.6 (a) and (b), where the linear regression of modelling $n$ data points is performed and each sparse biosignal is restored from noisy measurements (e.g., $n=1000, m=250$, and SNR $=25 \mathrm{~dB}$ ). Here, the simulation results show that the digital CS-ICA algorithms have fewer reconstruction errors than traditional digital CS methods, the reason for which is that the ICA algorithms can capture all $n$ data points containing the $K$ coefficients and make the biosignal detectable at a high SNR, whereas traditional CS methods cannot guarantee whether the derived measured samples (15), $m$, contain all non-zero components $K$, which are randomly distributed across the sparse vector $s_{i}$ and captured by increasing the number of measurements $m$.

Fig.6 (c) compares the performance of both methods in terms of the mean squared error (MSE) with standard (digital) ICA algorithms, where the CS-circuit components are omitted and the ICA scheme must perform full measurements to create $\mathrm{W}$, i.e., $m=1000$. The results indicate that the performance of CS-ICA algorithms can be close to standard ICA algorithms for medium and high SNR regimes, both of which are roughly equivalent at low SNR values. We can consequently conclude that the digital CSICA algorithms can significantly reduce the effect of motion artifacts and interference on recovery of sparse biosignals as observed in (17), where $\left\|v_{i}\right\|_{l_{2}} \leq \sigma_{i}^{2} /\left\|h_{i}\right\|^{2}$.

According to the central limit theorem and the law of large numbers, when adding a large number of independent random variables (i.e., the number of wearable biosensors gets larger, $N \rightarrow \infty$ ), the total tends to a Gaussian distribution even if the original variables (source biosignals) are not normally distributed. Since the CS methods have inherently Gaussian characteristics, they can be used instead of the ICA algorithms to retrieve sparse biosignals. Furthermore, the results demonstrate that the CS-ICA algorithms perform better than traditional CS methods when tested under the same conditions (e.g., $m=300$ ).

In general, Fig.6 results can be treated as benchmarks for the CS-ICA algorithms. On one hand, the ICA can increase the sparse biosignal strength received (by reducing 
$\mathrm{s}_{1}(\mathbf{t})$

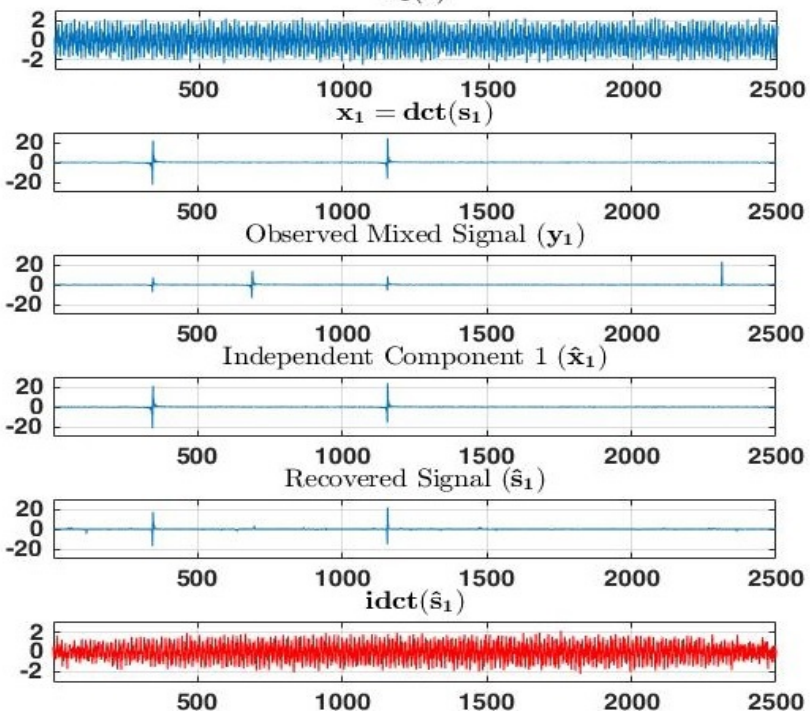

$\mathbf{s}_{2}(\mathbf{t})$
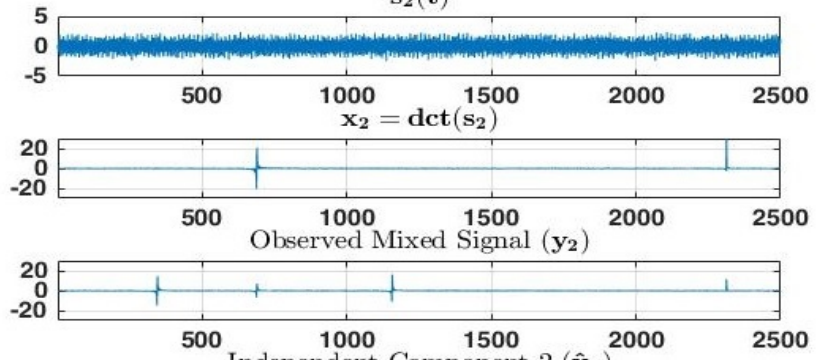

Independent Component $2\left(\hat{\mathbf{x}}_{2}\right)$
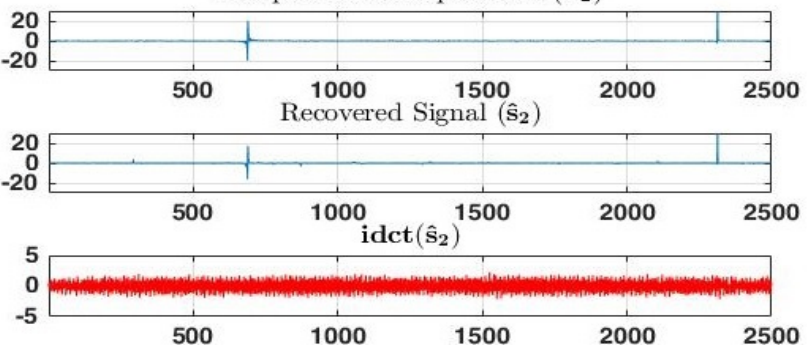

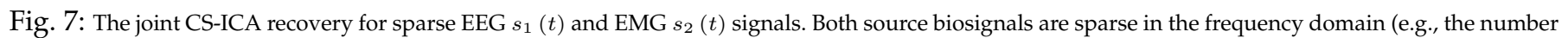
of samples per sparse biosignal is $n=2500$ ) and reconstructed in the time domain (e.g., $m=170$ ) at an SNR level of 30dB.

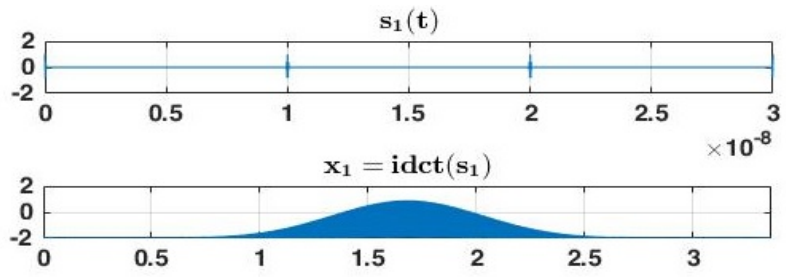

Observed Mixed Signal ( $\left.\mathbf{y}_{1}\right)$
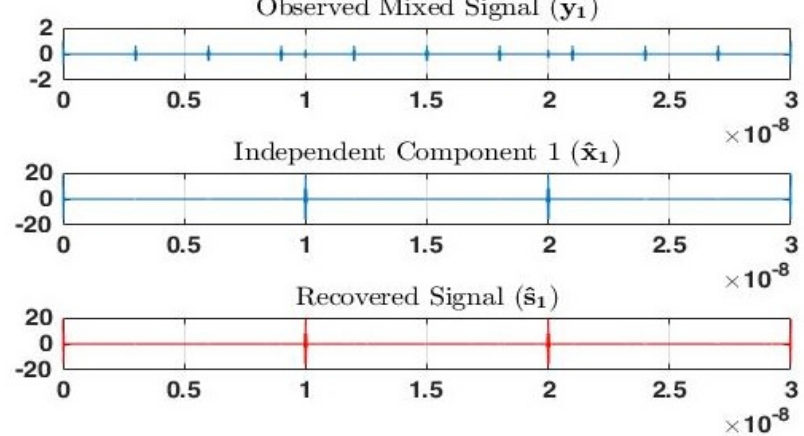
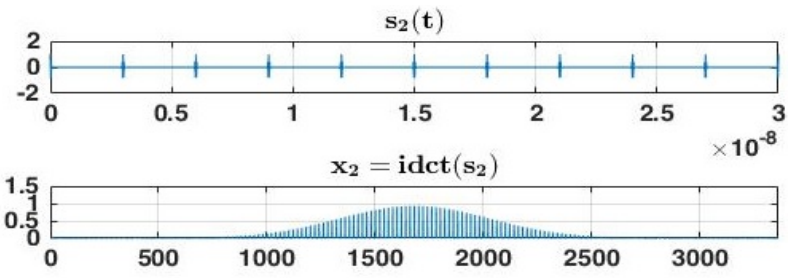

Observed Mixed Signal ( $\left.\mathbf{y}_{2}\right)$
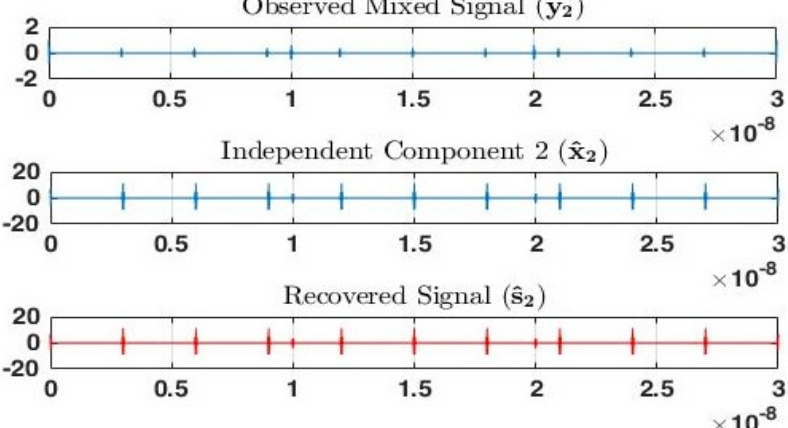

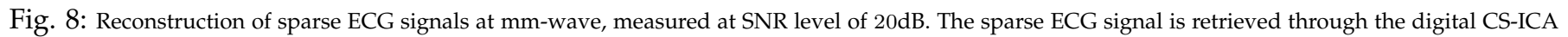

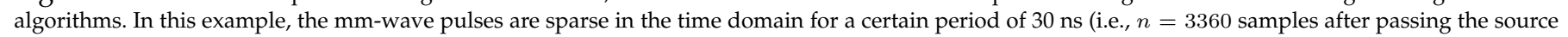
biosignal $s(t)$ through IDCT) and are recovered from just 300 snapshots.

motion artifacts and interference) and use low-resolution DACs to restore the sparse biosignal. On the other hand, the CS methods can reduce the sampling rate $f_{s}$ and make the DACs operate at low speed. This process is called an "exchange of interests" between CS and ICA methods.

\section{EXPERIMENTAL RESULTS}

Fig.7 shows the joint CS-ICA recovery for two sparse biosignals, e.g., EEG and electromyography (EMG) signals where $N=2, M=2$. Both biosignals are sparse in the frequency domain (e.g., the number of samples per source signal is $n=2500$ and the number of spikes is $K=2$ ) with various periods for each sparse biosignal (e.g., $T=1$ second for the sparse EEG signal $s_{1}(t)$ and $T=2$ seconds for the sparse EMG signal $s_{2}(t)$. The relatively few coefficients capture most of the signal energy, e.g., $m=170$, taken in the time domain. This example obviously shows the gain acquired by the digital CS-ICA algorithms, where the sparse biosignals can be restored in only a few measurements.

Fig. 8 compares the performance of CS-ICA algorithms and standard CS methods to two sparse ECG signals operating in the $28 \mathrm{GHz}$ band [35]. The mm-wave pulse train is sparse in the time domain, e.g., the pulse repetition period $(\triangle t)$ is $10 \mathrm{~ns}$ for the first sparse ECG signal and $3 \mathrm{~ns}$ for the second sparse ECG signal, $N=2$, where each sparse biosignal has the same frame size. For example, $T=30 \mathrm{~ns}$ for the sparse ECG signals $s_{1}(t)$ and $s_{2}(t)$. The reason is due to the assumption that the mm-wave signals are sparse in the time domain rather than the frequency domain to simplify the construction of the IDCT matrix $\Psi_{n \times n}$ (where $n$ is very large) required to produce the sparse source vectors 


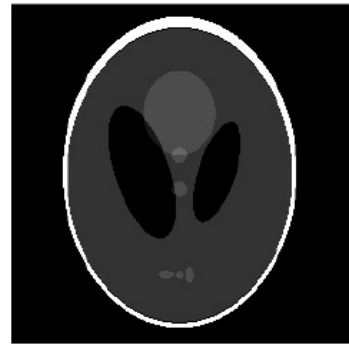

(a) Source image

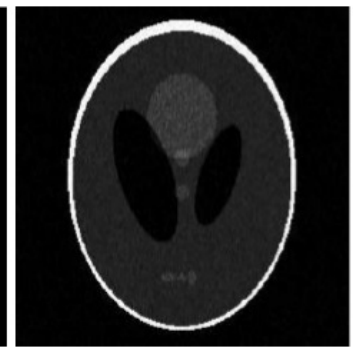

(b) Noisy image

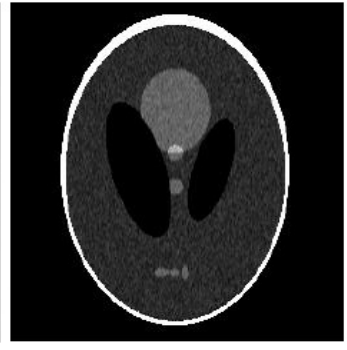

(c) Standard ICA recovery

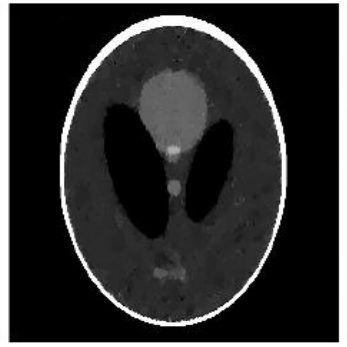

(d) Joint CS-ICA recovery

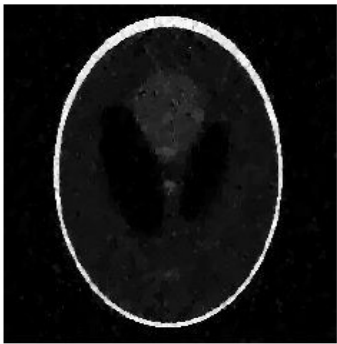

(e) Standard CS

Fig. 9: Phantom recovery experiments at SNR 15dB, where the CS-ICA recovery and standard CS methods restore the sparse MEG image at $n=65,536$ samples and $m=6213$ snapshots, while standard ICA methods perform accurate image recovery at $m=65,536$ snapshots.

$x_{1}(t)$ and $x_{2}(t)$. Here, the sampling rate is calculated as $f_{s}=4 \times 28$ giga samples/sec, and the total number of samples (data points) taken to perform a Fourier transform, is $n=3360$. Moreover, in this example, assume that the wearable ECG sensors have line-of-sight (LOS) paths with the data aggregator sensors $M=2$ (e.g., $S N R=20 \mathrm{~dB}$ ), where each sparse ECG signal is retrieved from a few measurements that are captured in the frequency domain (e.g., $f_{s}=4 \frac{m}{T}$, where $m=300$ ). In this setup, the mixing matrix elements of $\mathrm{H} \in R^{2 \times 2}$ are uniformly distributed and the unmixing matrix $\mathrm{W}=\mathrm{H}^{T}$ is calculated through the FastICA algorithms. Our experimental results show that the digital CS-ICA algorithms can provide a more accurate and rapid recovery of ECG signals compared to traditional CS methods.

Another practical example of real-time patient monitoring systems can be found in wearable magnetoencephalography (MEG) scanners that are used for mapping brain activity. Fig.9 illustrates a phantom recovery experiment that exactly mimics MEG images that can be contaminated with outside noise and motion artifacts. In this example, we restore a large image size of 256x256 pixels of noisy measurements (e.g., $S N R=15 \mathrm{~dB}$ ), where the biosignal of interest $s_{1}$ is extracted from an unwanted biosignal $s_{2}$ (e.g., $N=2, M=2$ ). The sparse MEG image is recovered using the min-total variation (TV) ${ }^{2}$ method [36], with 25 non-zero frequency components distributed uniformly in the Fourier domain, where a few measurements are created to restore the desired MEG image (e.g., $m=6213$ ). Compared with standard digital ICA methods, the CS-ICA recovery methods can reduce noise and number of measurements needed (e.g., from 65,536 to 6213 snapshots), allowing receivers to use low-speed/low-resolution DACs to retrieve the sparse source MEG signal. Furthermore, the experiential results show that the digital CS-ICA algorithm can achieve highresolution of MEG images compared to standard digital CS method. However, it should be noted that standard digital ICA algorithms have poor resolution of highly correlated brain sources.

\section{Conclusion}

In this paper, we present a new information-theoretic framework for CS-ICA algorithms that can address the sparse sig-

2. The min-total variation (TV) solution is used for the large-scale 2Dstructure instead of $\min -l_{1}$, which ensures stable recovery for sparse MEG images with high accuracy. nal recovery and acquisition problem in wearable biosensor networks. The proposed framework aims to determine the sampling rate required to recover sparse biosignals in edge computing devices. Using digital CS-ICA implementation, we can reduce the number of measurements, increase the noise robustness, and improve the accuracy and efficiency of standard digital CS methods. Our results showed that the CS-ICA algorithms can perform better than standard CS methods when a sparse biosignal contains motion artifacts and interference, where $K$-sparse biosignals/images are recovered when sampled below the Nyquist sampling rate $f_{s} \geq 2 \frac{m}{T}$, where $m \geq 2 \log _{2}\left(\frac{n}{K}\right) / \log _{2}(1+M . S N R)$ samples. Compared to standard ICA algorithms, the CSICA algorithms can reduce the sampling requirements for digital-to-analog converters as well as the computational complexity of ICA algorithms when working on noisy measurements while improving the quality of reconstructed sparse biosignals, which in turn reduces the power consumption of edge devices, and retrieve sparse biosignals in fewer measurements.

The proposed sensing approach will generally provide significant environmental and economic benefits to healthcare delivery systems, allowing clinicians to monitor patient conditions directly and lighten the burden of healthcare costs.

\section{REFERENCES}

[1] S. K. Khaitan and J. D. McCalley, "Design techniques and applications of cyberphysical systems: A survey," IEEE Syst. J., vol. 9, no. 2, pp. 350-365, Jun. 2015.

[2] T.-P. Jung, S. Makeig, M. Westerfield, J. Townsend, E. Courchesne, T. J. Sejnowski, "Removal of eye activity artifacts from visual event-related potentials in normal and clinical subjects," Clin. Neurophysiol., vol. 111, pp. 1745-1758, 2000.

[3] J. Lee et al., "Motion Artifact Reduction in Wearable Photoplethysmography Based on Multi-Channel Sensors with Multiple Wavelengths," Sensors, 20, 1493, 2020.

[4] F. T. Zuhra, K. B. A. Bakar, A. A. Arain, U. A. Khan and A. R. Bhangwar, "MIQoS-RP: Multi-Constraint Intra-BAN, QoS-Aware Routing Protocol for Wireless Body Sensor Networks,"IEEE Access, vol. 8, pp. 99880-99888, 2020.

[5] M.F.Shaik, V.L.N. Komanapalli and M.M. Subashini, "A Comparative Study of Interference and Mitigation Techniques in Wireless Body Area Networks," Wireless Pers Commun 98, 2333-2365, 2018.

[6] S. Movassaghi, A. Majidi, A. Jamalipour, D. Smith and M. Abolhasan, "Enabling interference-aware and energy-efficient coexistence of multiple wireless body area networks with unknown dynamics," IEEE Access, vol. 4, pp. 2935-2951, 2016.

[7] M. K. Hasan, M. Shahjalal, M. Z. Chowdhury, and Y. M. Jang, "Real-time healthcare data transmission for remote patient monitoring in patch-based hybrid OCC/BLE networks," Sensors, vol. 19, no. 5, pp. 1-23, Mar. 2019. 
[8] Y. C. Eldar "Sampling Theory: Beyond Bandlimited Systems," Cambridge University Press, Apr. 2015.

[9] E. Candes and M. Wakin, "An introduction to compressive sampling," IEEE Signal Process. Mag., vol. 25, no. 2, pp. 21-30, Mar. 2008.

[10] A. Hyvarinen and E. Oja. "Independent component analysis: algorithms and applications," Neural networks, 13(4-5):411-430, 2000.

[11] Z. Zhang et al., "Compressed sensing for energy-efficient wireless telemonitoring of noninvasive fetal ECG via block sparse Bayesian learning," IEEE Trans. Biomed. Eng., vol. 60, no. 2, pp. 300-309, Feb. 2013.

[12] H. Mamaghanian, N. Khaled, D. Atienza, and P. Vandergheynst, "Compressed sensing for real-time energy-efficient ECG compression on wireless body sensor nodes," IEEE Trans. on Biomed. Eng., vol. 58, no. 9, pp. 2456-2466, 2011.

[13] B. Liu, Z. Zhang, G. Xu, H. Fan, and Q. Fu, "Energy efficient telemonitoring of physiological signals via compressed sensing: A fast algorithm and power consumption evaluation," Biomed. Signal Proces., vol. 11, pp. 80-88, 2014.

[14] F. Chen, A. P. Chandrakasan, and V. Stojanovic, "Design and analysis of a hardware-efficient compressed sensing architecture for data compression in wireless sensors," IEEE J. Solid-State Circuits, vol. 47, pp. 744-756, Mar. 2012.

[15] D. Gangopadhyay, E. G. Allstot, A. M. R. Dixon, K. Natarajan, S. Gupta, and D. J. Allstot, "Compressed sensing analog front-end for bio-sensor applications," IEEE J. Solid-State Circuits, vol. 49, no. 2, pp. 426-438, Feb. 2014

[16] F. Pareschi, P. Albertini, G. Frattini, M. Mangia, R. Rovatti, and G. Setti, "Hardware-algorithms co-design and implementation of an analog-to-information converter for biosignals based on compressed sensing," IEEE Trans. Biomed. Circuits Syst., vol. 10, no. 1, pp. 149-162, Feb. 2016.

[17] K. K. Shyu, M. H. Lee, Y. T. Wu, and P. L. Lee, "Implementation of pipelined fastICA on FPGA for real-time blind source separation," IEEE Trans. Neural Netw., vol. 19, no. 6, pp. 958-970, Jun. 2008.

[18] L. Van, D. Wu and C. Chen, "Energy-Efficient FastICA Implementation for Biomedical Signal Separation," IEEE Trans. Neural Netw., vol. 22, no. 11, pp. 1809-1822, 2011.

[19] M. Stanaćević, S. Li, G. Cauwenberghs, "Micropower mixed-signal VLSI independent component analysis for gradient flow acoustic source separation," IEEE Trans. Circuits Syst. I Reg. Papers, vol. 63, no. 7, pp. 972-981, Jul. 2016

[20] S. Fauvel, A. Agarwal and R. Ward, "Compressed sensing and energy-aware independent component analysis for compression of EEG signals," 2013 IEEE International Conference on Acoustics, Speech and Signal Processing, Vancouver, BC, 2013, pp. 973-977.

[21] B. Zhou, X. Wu, Z. Lv, L. Zhang and C. Zhang, "Independent component analysis combined with compressed sensing for EEG compression in BCI," 2015 10th International Conference on Information, Communications and Signal Processing (ICICS), Singapore, 2015, pp. 1-4.

[22] B. Mijovic et al., "Independent Component Analysis as a Preprocessing Step for data Compression of Neonatal EEG," In Proceedings of the 33rd Annual International Conference of the IEEE Engineering in Medicine and Biology Society (EMBS '11), Boston, MA, USA, pp. 7316-7319, Aug. 2011.

[23] M. Stanaćević, S. Li, and G. Cauwenberghs, "Micropower mixedsignal VLSI independent component analysis for gradient ow acoustic source separation," IEEE Trans. Circuits Syst., vol. 63, no. 7, pp. 972-981, Jul. 2016.

[24] S. Fauvel and R. K. Ward, "An energy efficient compressed sensing framework for the compression of electroencephalogram signals," Sensors, vol. 14, no. 1, pp. 1474-1496, 2014.

[25] D. Kanemoto, S. Katsumata, M. Aihara and M. Ohki, "Framework of Applying Independent Component Analysis After Compressed Sensing for Electroencephalogram Signals," 2018 IEEE Biomedical Circuits and Systems Conference (BioCAS), Cleveland, OH, pp. 1-4, 2018.

[26] G. Da Poian, R. Bernardini and R. Rinaldo, "Separation and Analysis of Fetal-ECG Signals From Compressed Sensed Abdominal ECG Recordings," IEEE Trans. Biomed. Eng., vol. 63, no. 6, pp. 12691279, June 2016.

[27] S. KATSUMATA, D. KANEMOTO and M. OHKI, "Applying Outlier Detection and Independent Component Analysis for Compressed Sensing EEG Measurement Framework," 2019 IEEE Biomedical Circuits and Systems Conference (BioCAS), Nara, Japan, pp. 1-4, 2019

[28] f. Cao and N. Murata, "A stable and robust ICA algorithm based on t-distribution and generalized Gaussian distribution models," in Proc. IEEE Signal Process. Soc. Workshop on Neural Netw. Signal Process., Madison, WI, Aug. 1999, pp. 283-292.

[29] J. W. Choi, B. Shim, Y. Ding, B. Rao and D. I. Kim, "Compressed Sensing for Wireless Communications: Useful Tips and Tricks," IEEE Commun. Surv. Tutor., vol. 19, no. 3, pp. 1527-1550, Feb. 2017.

[30] E. J. Candès, "The restricted isometry property and its implications for compressed sensing," Compte Rendus de l'Academie des Sciences, vol. Serie I, no. 346, pp. 589-592, 2008.

[31] F. Marvasti et al., "A unified approach to sparse signal processing," EURASIP J. Adv. Signal Process., vol. 2012, no. 44, pp. 1-45, 2012.

[32] F. Renna, R. Calderbank, L. Carin, and M. R. D. Rodrigues, "Reconstruction of signals drawn from a Gaussian mixture via noisy compressive measurements," IEEE Trans. Signal Process., vol. 62, no. 9, pp. 2265-2277, May. 2014.

[33] T. M. Cover and J. A. Thomas, Elements of Information Theory. New York: Wiley, 1991.

[34] O. Rivasplata. "Subgaussian random variables: An expository note," Unpublished note, 2012.

[35] S. Suzuki, T. Matsui, B. Escalante, "Remote sensing for medical and health care applications," in Remote Sensing-Applications, Rijeka, Croatia: InTech, 2012.

[36] E. Candès, J. Romberg, and T. Tao, "Stable signal recovery from incomplete and inaccurate measurements," Comm. Pure Appl. Math., vol. 59, no. 8, pp. 1207-1223, Aug. 2006.

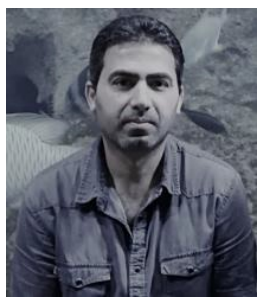

Yahia Alghorani (S'13-M'16) received his M.Sc. degree in computer engineering from University Kebangsaan Malaysia, Selangor, Malaysia, in 2007, and his Ph.D. degree in computer and software engineering from Ecole Polytechnique de Montreal, Montreal, QC, Canada, in 2015 From 2009 to 2010, he was an RF engineer with Batelco. From 2010 to 2012, he was a wireless networks engineer with Huawei. From 2017 to 2019, he was a research fellow at University College Dublin, Ireland. Dr. Alghorani is currently a research associate at Lakehead University, ON, Canada. His research interests include intelligent transportation systems, connected vehicles, artificial intelligence, machine learning, deep learning, medical wearables and e-health, statistical signal processing, and hardware/software co-design.

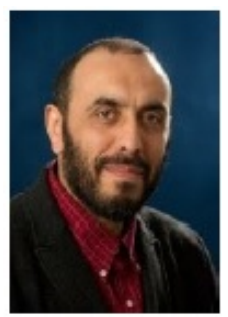

Salama S. Ikki received his Ph.D. degree in Electrical Engineering from Memorial University, St. Johns, NL, Canada, in 2009. From February 2009 to February 2010, he was a Postdoctoral Researcher at the University of Waterloo, ON, Canada. From February 2010 to December 2012, he was a Research Assistant with INRS, University of Quebec, Montreal, QC, Canada. $\mathrm{He}$ is currently an Associate Professor of wireless communications with Lakehead University, Thunder Bay, ON, Canada. He is the author of 100 journal and conference papers and has more than 4000 citations and an $\mathrm{H}$-index of 30 . His research interests include cooperative networks, multiple-input-multiple-output, spatial modulation, and wireless sensor networks. Dr. Ikki served on the Editorial Board of IEEE Communications Letters and Institution of Engineering and Technology Communications. He also served as a Technical Program Committee member for various conferences, including IEEE International Conference on Communications, IEEE Global Communications Conference, IEEE Wireless Communications and Networking Conference, IEEE Spring/Fall Vehicular Technology Conference, and IEEE International Symposium on Personal, Indoor, and Mobile Communications. Dr. Ikki received the Best Paper Award for his paper published in the EURASIP Journal on Advanced Signal Processing. He also received IEEE Communications Letters, IEEE Wireless Communications Letters, IEEE Transactions on Vehicular Technology and IEEE Transactions on Communications exemplary reviewer certificates for 2012, 2013 and 2014, respectively. 